\title{
MUSA VELUTINA (MUSACEAE): ASPECTOS ECOLÓGICOS QUE HAN FAVORECIDO SU DISPERSIÓN EN ECOSISTEMAS NATURALES
}

\section{MUSA VELUTINA (MUSACEAE): ENVIRONMENTAL CONSIDERATIONS THAT HAVE FAVORED ITS SPREAD IN NATURAL ECOSYSTEMS}

\author{
Brayan Morera Chacón ${ }^{1}$ \\ Jorge Eduardo Jiménez Castro ${ }^{2}$ \\ Peyton M. Steinbacher ${ }^{3}$
}

Recibido: 28.04 .19

Aprobado: 30.04 .20

DOI: $10.15517 /$ isucr.v21i43.41993

\begin{abstract}
Resumen
Musa velutina es una planta considerada una invasión biológica en tres distintas zonas de Costa Rica. Una de las características que ha facilitado su invasión es la utilidad que le da el ser humano, además de sus características fisiológicas. El trabajo se realizó en un parche de bosque secundario en el distrito de Los Ángeles en San Ramón. Se analizaron algunas características de la planta y determinamos que en Los Ángeles se pueden encontrar frutos con casi el doble de semillas con respecto a las plantas en su rango de distribución natural. También presentamos el análisis de tres técnicas de captura (observación directa, foto trampeo y redes de niebla) utilizadas para determinar los depredadores de M. velutina. Se logró determinar la existencia de seis especies depredadoras, en la cual la Guatusa es la especie con mayor número de capturas. Se recomienda utilizar estos métodos para encontrar sus depredadores en otros sitios donde se considera una especie invasora.
\end{abstract}

Palabras claves: Especie invasora; Depredadores; Dispersores; Técnicas de captura.

\footnotetext{
Abstract

Musa velutina is a plant considered a biological invasion in three different areas of Costa Rica. One feature that has facilitated its invasion is the utility that it gives humans such as ornamental decor,

${ }^{1}$ Estudiante de Bach. Gestión de los Recursos Naturales. Universidad de Costa Rica Sede Regional de Occidente, San Ramón, Alajuela, Costa Rica. Email: morera.b91@gmail.com

${ }^{2}$ Lic. Turismo Ecológico y estudiante de Bach. Gestión de los Recursos Naturales, Universidad de Costa Rica, Sede Regional de Occidente, San Ramón, Alajuela, Costa Rica. Email: K_2_oi@hotmail.com

${ }^{3}$ Estudiante de Bach. Ciencias Ambientales, Bach. Biología. California State University, Chico, California, Estados Unidos. Email: psteinbacher@mail.csuchico.edu
} 
as well as their physiological characteristics. This study was conducted in a patch of secondary forest in the district of Los Angeles in San Ramon, Costa Rica. Plant characteristics were analyzed and found to have almost doubled the amount of seeds per fruit in comparison to native species of M. velutina found in India. We also present the analysis of three capture techniques (direct observation, photo trapping and mist nets) used to determine the predators of M. velutina. It was possible to determine the existence of six predatory species, of which the Agouti was observed to have the highest number of catches. We recommend using these methods to further investigate other possible predators that can be found in differing areas where $M$. velutina is considered an invasive species. Further research is needed to understand how to control this highly invasive plant species found in Costa Rica.

Keywords: Invasive species; Predators; Scatterers; Capture techniques.

\section{Introducción}

Esta planta ha sido usada como ornamental debido a sus llamativos frutos color rosa (Rojas, Bermúdez y Jiménez, 2006) y en otros casos como alimento, utilizando la inflorescencia para hacer ensaladas o consumir cocinada (Kennedy, 2009). Una de las causas de la expansión de las musas en los trópicos y su aceptación universal han sido las características favorables del fruto, ya que es consumido por muchas especies, esto unido a la facilidad de su propagación vegetativa (Müller, 2000). Debido a los diferentes usos que se le ha encontrado a esta planta y sus características fisiológicas ha conseguido aumentar significativamente su distribución. En muchos lugares de Costa Rica se utiliza esta especie como ornamental, sin embargo al ser una especie exótica presenta muchas ventajas sobre las especies nativas ya que puede dispersarse libremente sin la presencia de un depredador natural. En Sarapiquí, San Ramón y San Vito se ha descrito como una especie invasora (Barquero 2005, Morera y Granados 2013 \& Quirós 2014).

En el distrito de los Ángeles de San Ramón se estimó una densidad de 0,954 individuos por metro cuadrado con una abundancia relativa del $76 \%$ y un índice de diversidad máxima de $(1,62=34 \%)$ en plantas de un metro o más de altura. (Morera y Granados, 2013), esto demuestra el avanzado estado de invasión de esta planta. Para que esta planta llegara a ser altamente invasiva debe existir un medio de transporte efectivo que le facilite su dispersión.

El objetivo de este trabajo fue determinar posibles diferencias en plantas y frutos con respecto a las mismas en su rango de distribución natural, además evaluar tres diferentes técnicas de captura para 
determinar cuales especies animales se alimentan de $M$. velutina en un sitio donde se considera invasora

\section{Metodología}

Descripción del sitio

El trabajo se realizó en la reserva privada del Hotel Villa Blanca (FIGURA 1) en Los Ángeles de San Ramón coordenadas $10^{\circ} 12^{\prime} 11.6^{\prime \prime} \mathrm{N}$ y 84²9’06.5” O a una altura aproximada de 1119 m.s.n.m. Según el sistema de clasificación de zonas de vida de Holdriedge, este sitio pertenece a un bosque muy húmedo pre montano. El sitio cuenta con un bosque secundario fragmentado con tres áreas bastante afectadas por M. velutina, además en sus bordes existen varios jardines lo que pudo funcionar como punto de partida para la introducción de esta especie exótica dentro de la reserva.

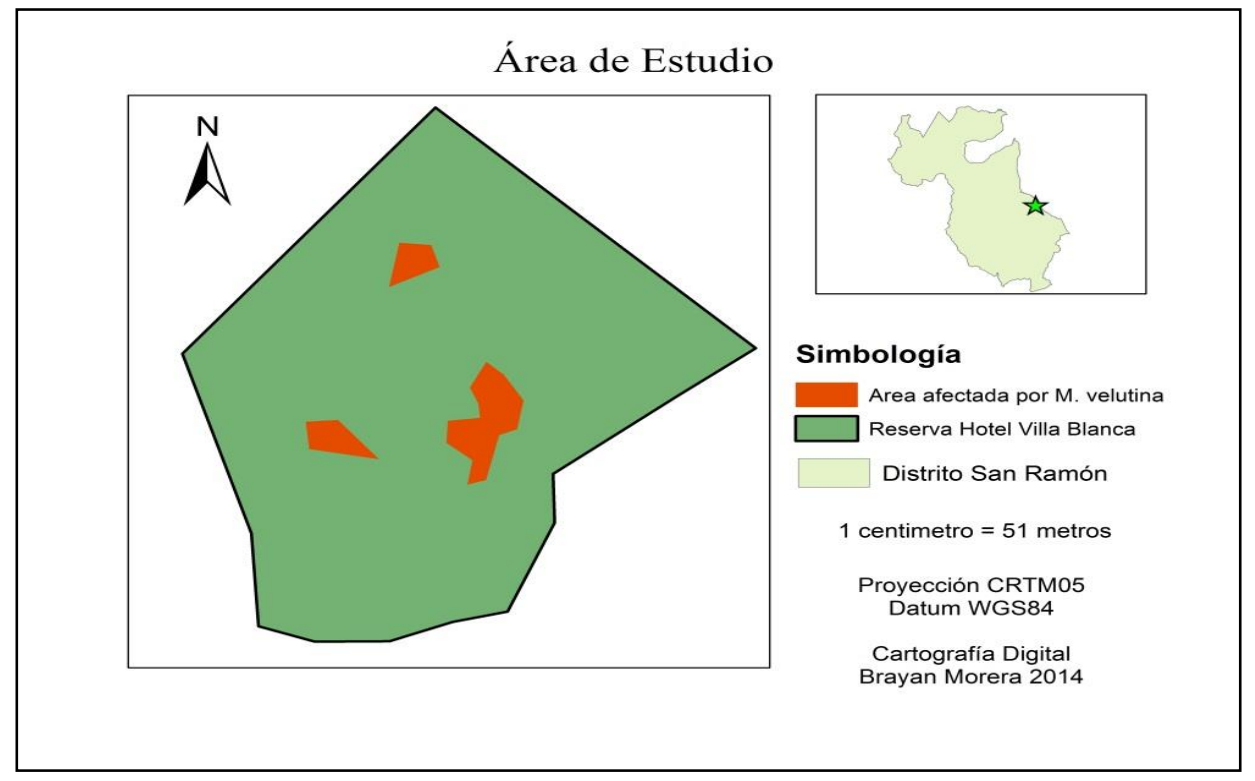

FIGURA 1. Área de estudio y zonas altamente afectadas por M. velutina.

Toma de datos en cuadriculas.

Se construyeron 25 cuadriculas de $100 \mathrm{~m}^{2}$ cada una en las zonas afectadas por M. velutina. Se consideraron como individuos aquellas plántulas que tuviesen flores o frutos. A cada individuo se le midió su tamaño total desde el suelo hasta la base del fruto o la flor. Se contó el número de frutos y se escogió un fruto al azar para medir su tamaño y contar sus semillas. 
Técnicas de captura para animales

Observación Directa

Se utilizaron los senderos ya existentes, los mismos se recorrieron aleatoriamente durante el día observando si algún animal ya fuera ave o mamífero se alimentaba del fruto de la M. velutina.

\section{Captura mediante Redes de Niebla}

Se colocaron redes de niebla a lo largo de los senderos extendiéndose a diferentes horas del día, en la mañana y tarde para capturar aves y en la noche para los murciélagos. Las redes fueron extendidas en los mayores picos de actividad tanto para aves como para murciélagos y en función de las condiciones ambientales, también se toman en cuenta sitios de transito potencial de especies, la cercanía con la Musa velutina y la facilidad para poder extenderlas. Las redes se colocaron a nivel del suelo las mismas tenían diferentes medidas ( $2.5 \mathrm{~m}$ de alto por $12 \mathrm{~m}$ de largo y $2.5 \mathrm{~m}$ de alto por $6 \mathrm{~m}$ de largo, todas con $20 \mathrm{~mm}$ de apertura de malla). Los murciélagos y aves capturados fueron puestos en una bolsa de tela y se dejaron un tiempo prudente para observar si excretaban, luego se tomaron las excretas y se llevaron al laboratorio para compararlas con las muestras de semillas de $M$ velutina ya procesadas para buscar similitudes. También se tomaron muestras de las excretas en el momento de ser capturados en la red ya que muchos de los individuos excretan ahí mismos. En esta técnica se considera como captura los individuos que presentaran evidencia de $M$. velutina en sus excretas.

\section{Foto trampeo}

Se contó con cuatro cámaras trampa MOULTRIE las cuales estuvieron monitoreando las 24 horas, las mismas fueron instaladas desde el 03 al 06 de junio del 2014, se ubicaron en sitios estratégicos donde abunda la Musa velutina, a una altura de $20 \mathrm{~cm}$ del suelo. Cerca de las cámaras se colocaron racimos de $M$ velutina ya maduros para que los posibles depredadores se alimentaran de ellos y así poder identificarlos. 
Análisis de datos

Se calculó el promedio para el tamaño de las plántulas, el número de frutos, el tamaño del fruto y el número de semillas. Se comparó los promedios, los valores máximos registrados y los valores reportados en la literatura.

Se calculó el índice de diversidad de Shannon en cada técnica de captura para determinar cual es más efectiva en la determinación de los depredadores de la planta.

\section{Resultados}

Al tomar las medidas de tamaño de la planta, cantidad de frutos, tamaño del fruto y cantidad de semillas para 510 individuos de $M$. velutina se observa que esos valores son muy similares a los reportados en la literatura, sin embargo la cantidad de semillas obtenidas es mucho mayor a lo reportado. El cuadro uno muestra los promedios y valores máximos obtenidos y se comparan con lo reportado en la literatura.

CUADRO 1. Comparación de los promedios y valores máximos obtenidos con respecto a los valores reportados en la literatura.

\begin{tabular}{|l|l|l|l|l|}
\hline & $\begin{array}{l}\text { Tamaño de la planta } \\
(\mathrm{cm})\end{array}$ & $\begin{array}{l}\text { Número de } \\
\text { frutos }\end{array}$ & $\begin{array}{l}\text { Tamaño del } \\
\text { fruto }(\mathrm{cm})\end{array}$ & $\begin{array}{l}\text { Número de } \\
\text { semillas }\end{array}$ \\
\hline $\begin{array}{l}\text { Promedios } \\
\text { obtenidos }\end{array}$ & 94,02 & 6 & 5,49 & 39 \\
\hline $\begin{array}{l}\text { Máximos } \\
\text { obtenidos }\end{array}$ & 147 & 23 & 9,7 & 143 \\
\hline $\begin{array}{l}\text { Reportado en } \\
\text { literatura }\end{array}$ & 150 & $20-30$ & $6-10$ & $70-80$ \\
\hline
\end{tabular}

Las capturas arrojaron como resultado un total de 21 individuos capturados de 6 especies diferentes, 3 mamíferos; Guatuza (Dasyprocta punctata), Pizote (Nasua narica) y un Ratón (Rodentia) no identificado, 3 aves; Yigüirro (Turdus grayi), Tangara común (Chlorospingus flavopectus), Saltón cabecicastaño (Arremon brunneinucha). De estas, 16 capturas se hicieron con cámaras trampa, 5 con observación directa y 0 con redes de niebla. Del total de capturas realizadas con los diferentes métodos la especie más capturada fue la Guatuza con un total de 12 capturas, después el pizote con 4, Yigüirro y la tangara común 2, el saltón cabecicastaño y el ratón con 1 captura El total de horas de muestreo fue de 121, 96 con cámaras trampa, 6 con observación directa 
y 19 con redes de niebla. El cuadro dos muestra el total de horas de muestreo, el número de capturas por método y por especie además de las especies registradas por técnica de captura.

CUADRO 2. Comparación de horas de muestreo total y numero de dispersores encontrados por técnica de captura.

\begin{tabular}{|c|c|c|c|c|}
\hline $\begin{array}{l}\text { Método de } \\
\text { captura }\end{array}$ & $\begin{array}{l}\text { Horas de } \\
\text { muestreo }\end{array}$ & $\begin{array}{l}\text { Numero de capturas u } \\
\text { observaciones por } \\
\text { método }\end{array}$ & $\begin{array}{l}\text { Especies } \\
\text { capturadas }\end{array}$ & $\begin{array}{l}\text { Numero de capturas u } \\
\text { observaciones por } \\
\text { especie }\end{array}$ \\
\hline \multirow{3}{*}{$\begin{array}{l}\text { Cámaras } \\
\text { trampa }\end{array}$} & \multirow{3}{*}{96} & \multirow{3}{*}{ 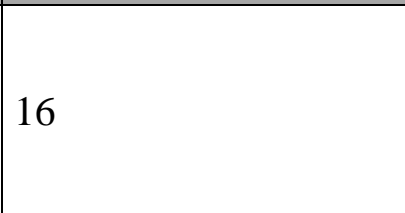 } & \begin{tabular}{|l} 
Dasyprocta \\
punctata
\end{tabular} & 12 \\
\hline & & & Nasua narica & 3 \\
\hline & & & Rodentia & 1 \\
\hline \multirow{4}{*}{$\begin{array}{l}\text { Observación } \\
\text { directa }\end{array}$} & \multirow{4}{*}{6} & \multirow{4}{*}{5} & Nasua narica & 1 \\
\hline & & & Turdus grayi & 2 \\
\hline & & & $\begin{array}{l}\text { Chlorospingus } \\
\text { flavopectus }\end{array}$ & 1 \\
\hline & & & $\begin{array}{l}\text { Arremon } \\
\text { brunneinucha }\end{array}$ & 1 \\
\hline $\begin{array}{l}\text { Redes } \\
\text { niebla }\end{array}$ & 19 & 0 & - & 0 \\
\hline Total & 121 & 21 & 6 & 21 \\
\hline
\end{tabular}

Para evaluar las técnicas de capturas se utilizó el índice de diversidad de Shannon, lo que significa que a una mayor diversidad la técnica es más efectiva para determinar las especies que se alimentan de $M$. velutina. Para las tres técnicas utilizadas la más efectiva fue la observación directa $(H=$ $1,921)$ seguido de las cámaras trampa $(H=1,014)$. En el caso de las redes de niebla no se evaluó porque no se obtuvieron capturas.

\section{Discusión}

En el género Musa todas las partes de la planta son útiles, incluidas las frutas de mala muerte, inflorescencia, hoja, pseudotallo, cormos y rizomas; en ellos se encuentra alimento, forraje, medicina, materiales y refugio domésticos. Las plantas también tienen ritual y significado ceremonial (Kennedy 2009). Simmonds (1962) describe las especies silvestres de Musa como malas hierbas de la selva de hábitats alterados, tales como taludes, laderas inestables o espacios forestales. La perturbación es una parte integral del ciclo de crecimiento dinámico de los bosques, proporcionando nichos para las plantas del sotobosque como musáceas (Argent 1979, Whitmore 
1984). Musa velutina ha sido utilizada por muchas culturas en campos como el gastronómico, paisajístico y de la salud, esto ha causado que la planta se allá propagado a nivel mundial, su dispersión se ha favorecido gracias a aspectos propios de su genética ya que como lo cita Sheela (2008), este tipo de plantas se adaptan muy bien a condiciones tropicales húmedas, que toleran el sol intenso y también condiciones de sombra parcial. Se propaga en suelos húmedos y por pseudotallos, además su néctar es apreciado por diferentes animales (Morera \& Umaña 1995).

Al ser una especie con alto potencial ornamental se conoce mucho acerca de su reproducción y de los cuidados que ocupa así como de las plagas que la atacan (Baskin \& Baskin 2001). Sin embargo es poco estudiado el efecto de esta planta sobre la biodiversidad local; ya que al ser una especie introducida puede convertirse en competidora para las locales por espacio o por dispersores. Este problema se puede extender dependiendo de las condiciones ambientales presentes en el ecosistema hasta convertirse en especie invasora. Es por este motivo que se hace urgente no solo conocer más acerca de la reproducción de la especie sino también que animales la dispersan y la depredan, para establecer estrategias de manejo y control.

Los valores máximos obtenidos de tamaño de la planta, numero de frutos y tamaño del fruto se aproximan bastante con lo reportado por Mamiyil et al (2013). Sin embargo la única diferencia que se puede observar entre las plantas nativas y las de San Ramón es la cantidad de semillas. Con un máximo de 140 semillas, casi el doble de las que se puede encontrar en las plantas con rangos de distribución natural, se puede observar una característica que posiblemente colabora a que la especie sea invasora cuando una planta esta produciendo más semillas de lo normal.

Como no solo basta una gran cantidad de semillas consideramos que especies nativas están brindando ese servicio de transporte a la planta, ya que a la vez estas especies obtienen alimento que es fácil de capturar y abundante, formándose una interacción mutualista que incorpora la nueva planta al ecosistema. Esas interacciones cumplen un papel importante en los procesos de invasión ya que pueden facilitar la dispersión de semillas de especies exóticas (Richardson et al. 2000).

Uno de los principales aportes de esta investigación es la determinación de seis especies depredadoras de M. velutina (cuadro2). Según Garmendia \& Samo (2005), los métodos de captura 
están basados en la caza y posterior liberación de animales después de un periodo determinado de tiempo; cada técnica tiene sus ventajas y desventajas y no se pueden aplicar las mismas técnicas a todos los organismos; es por este motivo que se utilizaron diferentes técnicas para grupos diferentes de animales. Para mamíferos voladores y aves se utilizaron las redes de niebla ya que son el método de captura más común y barato (Kunz \& Kurta 1988), además para intensificar el esfuerzo de muestreo se utilizó el método de observación directa que consiste en la recopilación de datos observando y contando los individuos de interés en un punto específico en un periodo de tiempo determinado, este método es muy utilizado en casos donde no se requieran mediciones de los individuos (Scheaffer et al 2007); para la investigación este método fue muy útil puesto que nosotros solo necesitábamos identificar especies no medirlas, este método fue el más efectivo ya que logró determinar el mayor diversidad de especies depredadoras. Por último y para maximizar el tiempo de observación se utilizó el método de foto trampeo o cámaras trampa, este último método fue el que más número de capturas obtuvo pero también fue el que más horas de muestreo acumuló (cuadro 2).

La Guatusa fue la especie más capturada siendo el depredador más frecuente de esta planta invasora, las guatusas se caracterizan por su patrón disperso de enterrar las semillas, suelen enterrarlas en distintos lugares, generalmente a una profundidad de $2-4 \mathrm{~cm}$ en el suelo y en la hojarasca (Fung, 2011). Suelen comer en sitios oscuros y llevan sus frutas y semillas a comederos bien protegidos (Smythe 1991). Esta especie podría ser un perfecto dispersor de M. velutina, sin embargo se necesita estudiar la germinación de esas semillas.

Otro aporte que deja la investigación es la comparación de métodos para saber cual es mejor utilizar. Se recomienda utilizar la observación directa porque se obtuvo una mayor diversidad, sin embargo se debe complementar con otras técnicas para grupos animales difíciles de observar. Galindo (1998) considera que los animales son muy importantes en la reproducción, colonización y establecimiento de especies de plantas. Una de las piezas faltantes para entender más la invasión de $M$. velutina es conocer cuales son los depredadores son efectivamente dispersores y pretendemos que este trabajo sirva de base para el diseño experimental de futuras investigaciones. 


\section{Conclusiones}

1. En San Ramón es posible encontrar frutos con casi el doble de semillas con respecto a las plantas nativas.

2. Se reportaron seis especies de animales que depredan $M$. velutina, sin embargo no se pueden determinar como dispersoras hasta estudiar la germinación de las semillas que transportan.

3. La observación directa es la técnica que logró capturar la mayor diversidad de especies por lo que se recomienda para utilizarla en la determinación de depredadores siempre y cuando también se complemente con otras técnicas.

\section{Bibliografía}

Argent, G. (1979). New Guinea bananas a problematic genetic resource. Pg. 57-70 in Biological Aspects of Plant Genetic Resource Conservation in South-East Asia.

Barquero, K. (2005). Musa velutina en La Selva: dispersión de una especie exótica. Ecología Tropical y Conservación 1 (2): 184-186.

Baskin, C.; Baskin, J. M. (2001). Seeds: Ecology, Biogeography, and Evolution of Dormancy and Germination. California, USA: Academic Press, Elsevier Pg. 666.

Fung, E.M. (2011). Dispersión de semillas por mamíferos terrestres en bosques latifoliados del Atlántico Norte de Nicaragua después del huracán Félix. Tesis de Maestría. CATIE. Disponible en orton.catie.ac.cr/repdoc/A8952E/A8952E.PDF

Galindo, G. J. (1998). Dispersión de semillas por murciélagos: Su importancia en la regeneración y conservación del bosque tropical. Acta zool. Mex (n.s.) 73: 5774. Disponible en wwwl.inecol.edu.mx/azm/documentos/73/73d-Galindo.pdf

Garmendia, A.; Samo , A.J.; (2005). Prácticas de Ecología. Valencia, España: Editorial Universidad Politécnica de Valencia. Págs.174.

Kennedy J. (2009). Bananas and people in the homeland of genus Musa: not just pretty fruit. Ethnobotany Research and Applications 7:179-197. Disponible en http://scholarspace.manoa.hawaii.edu/handle/10125/12514

Kunz, T.H.; Kurta, A. (1988). Capture methods and holding devices. In: Ecological and Behavioral Methods for the Study of Bats (ed. T.H. Kunz). Washington DC: Smithsonian Institution pg. 1-29. 
Mamiyil, S. A. B. U., Joe, A., y Sreejith, P. E. (2013). Musa velutina subsp. markkuana (Musaceae): a new subspecies from northeastern India. Phytotaxa, 92(2), 49-54. Disponible en http://biotaxa.org/Phytotaxa/article/view/phytotaxa.92.2.3

Morera, B. y Granados, A. (2013). Distribución y abundancia de Musa velutina en un bosque nuboso, Ángeles de San Ramón, Alajuela, Costa Rica. Red Latinoamericana para el Estudio de Especies Invasoras 3: 16-22. Disponible en http://www.institutohorus.org.br/RLEPI/boletim_RLEPI_v3_n1_2013.pdf

Morera, J.; Umaña, C. (1995). Programa Agricultura Tropical Sostenible Unidad Recursos Filogenéticos. Turrialba, Costa Rica: Jardín Botánico Cabiria-7. CATIE Pg. 34. Disponible en orton.catie.ac.cr/repdoc/A4180E/A4180E.PDF

Müller, L. (2000). Manual de Laboratorio de Morfología Vegetal. Turrialba, Costa Rica: CATIE $259 \mathrm{pg}$.

Quirós, R. (2014). Encargado de Investigación. OTS. Las Cruces. Comunicación personal. Richardson, D. M.; Allsopp, N.; D'Antonio, C. M.; Milton, S. J., y Rejmanek, M. (2000).

Plant invasions - the role of mutualisms. Biological Reviews, 75(1), $\quad 65-93$.

Disponible en http://www.ncbi.nlm.nih.gov/pubmed/10740893

Rojas, F.; Bermúdez, G. y Jiménez, Q. (2006). Plantas Ornamentales del Trópico. Cartago, Costa Rica: $\quad$ Editorial Tecnológica de Costa Rica.

Scheaffer, R. L.; Mendenhall, W.; Ott, L. (2007). Elementary Survey Sampling. 6 ${ }^{\mathrm{a}}$ ed. International . Madrid, España: Thomson editores Paraninfo S.A Pg. 462.

Sheela, V.L. (2008). Flowers for Trade. New India Publishing Agency. New Delhi, India. Págs. 369.

Simmonds, N.W. 1962. The Evolution of the Bananas. Longmans, Green, London.

Smythe, N. (1991). Dasyprocta punctate and Agouti paca(Guatusa, Cherenga, Agouti, Tepezcuintle, Paca). Pg 463-465 . In Historia Natural de Costa Rica. Eds. Janzen, D. y 174 colaboradores. San José, Costa Rica: Editorial de la Universidad de Costa Rica. 822 Pg.

Whitmore, T.C. (1984). Tropical Rain Forests of the Far East. Second edition, Oxford University Press, Oxford. 